\title{
Tendency towards Maximum Complexity in a Non-equilibrium Isolated System
}

\author{
Xavier Calbet \\ Instituto de Astrofísica de Canarias \\ Vía Láctea, $s / n$, \\ E-38200 La Laguna, Tenerife, Spain \\ E-mail: xca@ll.iac.es \\ Ricardo López-Ruiz \\ Departamento de Física Teórica \\ Facultad de Ciencias, Edificio A, Universidad de Zaragoza, \\ E-50009 Zaragoza, Spain \\ E-mail: rilopez@posta.unizar.es
}

(June 9, 2018)

\begin{abstract}
The time evolution equations of a simplified isolated ideal gas, the "tetrahedral" gas, are derived. The dynamical behavior of LMC (López, Mancini, Calbet) complexity is studied in this system. In general, it is shown that the complexity remains within the bounds of minimum and maximum complexity. We find that there are certain restrictions when the isolated "tetrahedral" gas evolves towards equilibrium. In addition to the well-known increase in entropy, the quantity called disequilibrium decreases monotonically with time. Furthermore, the trajectories of the system in phase space approach the max-
\end{abstract}


imum complexity path as it evolves toward equilibrium.

Typeset using REVTEX 


\section{INTRODUCTION}

Several definitions of complexity, in the general sense of the term, have been presented in the literature. These can be classified according to their calculation procedure into two broad and loosely defined groups.

One of these groups is based on computational science and consists of all definitions based on algorithms or automata to derive the complexity. Examples are the logical depth [1], the $\epsilon$-machine complexity [2], and algorithmic complexity [3]. These definitions have been shown to be very useful in describing symbolic dynamics of chaotic maps, but they have the disadvantage of being very difficult to calculate.

Another broad group consists of those complexities based on the measure of entropy or entropy rate. Among these, we may cite the metric or K-S entropy rate [4, 5], the thermodynamic depth [6], the effective measure complexity [7], and the simple measure for complexity [8]. These definitions have also been very useful in describing symbolic dynamical maps, the latter having been applied to a non-equilibrium Fermi gas [9]. They suffer the disadvantage of either being very difficult to calculate or having a simple relation to the regular entropy.

New definition types of complexity have recently been introduced. These are based on quantities that can be calculated directly from the distribution function describing the system. One of these is based on "meta-statistics" [10] and the other on the notion of "disequilibrium" 11. This latter definition will be referred to hereafter as the LMC complexity. These definitions, together with the simple measure for complexity [8] described above, have the great advantage of allowing easy calculations within the context of kinetic theory and of permitting their evaluation in a natural way in terms of statistical mechanics.

The disequilibrium-based complexity is easy to calculate and shows some interesting properties [11], but suffers from the main drawback of not being very well behaved as the system size increases, or equivalently, as the distribution function becomes continuous [12]. Feldman and Crutchfield tried to solve this problem by defining another equivalent term for disequilibrium, but ended up with a complexity that was a trivial function of the entropy. 
Whether these definitions of complexity are useful in non-equilibrium thermodynamics will depend on how they behave as a function of time. There is a general belief that, although the second law of thermodynamics requires average entropy (or disorder) to increase, this does not in any way forbid local order from arising [13. The clearest example is seen with life, which can continue to exist and grow in an isolated system for as long as internal resources last. In other words, in an isolated system the entropy must increase, but it should be possible, under certain circumstances, for the complexity to increase.

In this paper we will examine how LMC complexity evolves with time in an isolated system and we will show that it indeed has some interesting properties. The disequilibriumbased complexity defined in Ref. [11] actually tends to be maximal as the entropy increases in a Boltzmann integro-differential equation for a simplified gas.

In Sec. II LMC complexity definition is reviewed. We proceed to calculate the distributions which maximize and minimize the complexity and its asymptotic behavior, and also introduce the basic concepts underlying the time evolution of LMC complexity in Sec. III. Later, in Sec. [V], by means of numerical computations following a restricted version of the Boltzmann equation, we apply this to a special system, which we shall term "tetrahedral gas". Finally, in Sec. $\nabla$, the results and possible future lines of investigation are discussed, together with their possible applications. In the Appendix is given an analytical and a numerical demonstrations of the results of the numerical calculations for the tetrahedral gas.

\section{DEFINITION OF COMPLEXITY}

The definition of LMC complexity, $C$, is

$$
C=D \cdot H
$$

where $D$ is the disequilibrium term and $H$ is the entropy.

We assume that the system can be in one of $N$ possible accessible states, $i$. The probability of the system being in state $i$ will be given by the discrete distribution function, $f_{i}$. 
The system is defined such that, if isolated, it will reach equilibrium, with all the states having equal probability, $f_{\mathrm{e}}$.

These definitions imply that all values of $f_{i}$ are positive,

$$
f_{i} \geq 0
$$

and that a normalization, $I$, must hold such that

$$
I \equiv \sum_{i=1}^{N} f_{i}=1,
$$

and the equilibrium distribution function is,

$$
f_{\mathrm{e}}=\frac{1}{N}
$$

The definition of disequilibrium, $D$, is given as a distance to the probability in equilibrium, $f_{\mathrm{e}}$ :

$$
D \equiv \sum_{i=1}^{N}\left(f_{i}-f_{\mathrm{e}}\right)^{2} .
$$

The normalized entropy, $H$, is defined as

$$
H \equiv-\frac{1}{\ln N} \sum_{i=1}^{N} f_{i} \ln f_{i} .
$$

Note that since $0 \leq H \leq 1$ and $0 \leq D \leq(N-1) / N$, the complexity, $C$, is normalized $(0 \leq C \leq 1)$

\section{COMPLEXITY VERSUS TIME}

\section{A. Complexity versus entropy}

We are interested in an isolated system with an initial arbitrary discrete distribution, and which evolves toward equilibrium, in which it reaches an equiprobability distribution.

To study the time evolution of the complexity, a diagram of $C$ versus time, $t$, can be used. But, as we know, the second law of thermodynamics states that the entropy grows monotonically with time; that is, 


$$
\frac{d H}{d t} \geq 0
$$

This implies that an equivalent way to study the time evolution of the complexity can be obtained by plotting $C$ versus $H$. In this way, the entropy substitutes the time axis, since the former increases monotonically with the latter. The conversion from $C$ vs. $H$ to $C$ vs. $t$ diagrams is achieved by stretching or shrinking the entropy axis according to its time evolution. This method is a key point in all this discussion. Note that, in any case, the relationship of $H$ versus $t$ will, in general, not be a simple one 14.

\section{B. Maximum and minimum complexity}

When an isolated system evolves with time, the complexity cannot have any possible value in a $C$ versus $H$ map, but it must stay within certain bounds, $C_{\max }$ and $C_{\min }$. These are the maximum and minimum values of $C$ for a given $H$. Since $C=D \cdot H$, finding the extrema of $C$ for constant $H$ is equivalent to finding the extrema of $D$.

There are two restrictions on $D$ : the normalization, $I$, and the fixed value of the entropy, $H$. To find these extrema undetermined Lagrange multipliers are used. Differentiating Eqs. (3), (5) and (6) we obtain,

$$
\begin{aligned}
& \frac{\partial D}{\partial f_{j}}=2\left(f_{j}-f_{\mathrm{e}}\right), \\
& \frac{\partial I}{\partial f_{j}}=1, \\
& \frac{\partial H}{\partial f_{j}}=-\frac{1}{\ln N}\left(\ln f_{j}+1\right) .
\end{aligned}
$$

Defining $\lambda_{1}$ and $\lambda_{2}$ as the Lagrange multipliers, we get:

$$
2\left(f_{j}-f_{\mathrm{e}}\right)+\lambda_{1}+\lambda_{2}\left(\ln f_{j}+1\right) / \ln N=0 .
$$

Two new parameters, $\alpha$ and $\beta$, which are a linear combinations of the Lagrange multipliers are defined:

$$
f_{j}+\alpha \ln f_{j}+\beta=0
$$


where the solutions of this equation, $f_{j}$, are the values that minimize or maximize the disequilibrium.

In the maximum complexity case there are two solutions, $f_{j}$, to Eq. (12) which are shown in Table $\llbracket$. One of these solutions, $f_{\max }$, is given by

$$
H=-\frac{1}{\ln N}\left[f_{\max } \ln f_{\max }+\left(1-f_{\max }\right) \ln \left(\frac{1-f_{\max }}{N-1}\right)\right],
$$

and the other solution by $\left(1-f_{\max }\right) /(N-1)$.

The maximum disequilibrium, $D_{\max }$, for a fixed $H$ is

$$
D_{\max }=\left(f_{\max }-f_{\mathrm{e}}\right)^{2}+(N-1)\left(\frac{1-f_{\max }}{N-1}-f_{\mathrm{e}}\right)^{2},
$$

and thus, the maximum complexity, which depends only on $H$, is

$$
C_{\max }(H)=D_{\max } \cdot H \text {. }
$$

Note that the behavior of the maximum value of complexity versus $\ln N$ has been studied in Ref. [15].

Equivalently, the values, $f_{j}$, that give a minimum complexity are shown in Table [1]. One of the solutions, $f_{\min }$, is given by

$$
H=-\frac{1}{\ln N}\left[f_{\min } \ln f_{\min }+\left(1-f_{\min }\right) \ln \left(\frac{1-f_{\min }}{N-n-1}\right)\right],
$$

where $n$ is the number of states with $f_{j}=0$ and takes a value in the range $n=0,1, \ldots, N-2$.

The resulting minimum disequilibrium, $D_{\min }$, for a given $H$ is,

$$
D_{\min }=\left(f_{\min }-f_{\mathrm{e}}\right)^{2}+(N-n-1)\left(\frac{1-f_{\min }}{N-n-1}-f_{\mathrm{e}}\right)^{2}+n f_{\mathrm{e}}^{2} .
$$

Note that in this case $f_{j}=0$ is an additional hidden solution that stems from the positive restriction in the $f_{i}$ values, Eq. (2). To obtain these solutions explicitly we can define $x_{i}$ such that

$$
f_{i} \equiv x_{i}{ }^{2}
$$


These $x_{i}$ values do not have the restriction imposed by Eq. (2) and can take a positive or negative value. If we repeat the Lagrange multiplier method with these new variables a new solution arises: $x_{j}=0$, or equivalently, $f_{j}=0$.

The resulting minimum complexity, which again only depends on $H$, is

$$
C_{\min }(H)=D_{\min } \cdot H
$$

As an example, the maximum and minimum of complexity, $C_{\max }$ and $C_{\min }$, are plotted as a function of the entropy, $H$, in Fig. 1 for $N=4$. In Fig. 8 the maximum and minimum disequilibrium, $D_{\max }$ and $D_{\min }$, versus $H$ are also shown.

\section{Minimum "envelope"}

As shown in Fig. 2 the minimum disequilibrium function is piecewise defined, having several points where its derivative is discontinuous. Each of these function pieces corresponds to a different value of $n$ (Table [I). In some circumstances it might be helpful to work with the "envelope" of the minimum disequilibrium function. The function, $D_{\text {minenv }}$, that traverses all the discontinuous derivative points in the $D_{\text {min }}$ versus $H$ plot is

$$
D_{\text {minenv }}=e^{-H \ln N}-\frac{1}{N}
$$

and is also shown in Figure 2.

\section{Asymptotic behavior of the complexity as $N \rightarrow \infty$}

When $N$ tends toward infinity the probability, $f_{\max }$, of the dominant state has a linear dependence with the entropy,

$$
\lim _{N \rightarrow \infty} f_{\max }=1-H
$$

and thus the maximum disequilibrium scales as

$$
\lim _{N \rightarrow \infty} D_{\max }=(1-H)^{2} .
$$


The maximum complexity tends to

$$
\lim _{N \rightarrow \infty} C_{\max }=H \cdot(1-H)^{2}
$$

The limit of the minimum disequilibrium and complexity vanishes,

$$
\lim _{N \rightarrow \infty} D_{\text {minenv }}=0
$$

and thus

$$
\lim _{N \rightarrow \infty} C_{\min }=0
$$

In general, in the limit $N \rightarrow \infty$, the complexity is not a trivial function of the entropy, in the sense that for a given $H$ there exists a range of complexities between 0 and $C_{\max }$ (Eqs. 25] and [23]).

In particular, in this asymptotic limit, the maximum of $C_{\max }$ is found when $H=1 / 3$, or equivalently $f_{\max }=2 / 3$, which gives a maximum of the maximum complexity of $C_{\max }=$ 4/27. This was numerically calculated by Anteneodo and Plastino in Ref. [15].

\section{AN EXAMPLE: THE TETRAHEDRAL GAS}

\section{A. The tetrahedral gas}

We present a simplified example of an ideal gas: the tetrahedral gas. This system is generated by a simplification of the Boltzmann integro-differential equation of an ideal gas. We are interested in studying the disequilibrium time evolution.

The Boltzmann integro-differential equation of an ideal gas with no external forces and no spatial gradients is

$$
\frac{\partial f(\mathbf{v} ; t)}{\partial t}=\int d^{3} \mathbf{v}_{*} \int d \Omega_{\text {c.m. }} \sigma\left(\mathbf{v}_{*}-\mathbf{v} \rightarrow \mathbf{v}_{*}^{\prime}-\mathbf{v}^{\prime}\right)\left|\mathbf{v}_{*}-\mathbf{v}\right|\left[f\left(\mathbf{v}_{*}^{\prime} ; t\right) f\left(\mathbf{v}^{\prime} ; t\right)-f\left(\mathbf{v}_{*} ; t\right) f(\mathbf{v} ; t)\right],
$$


where $\sigma$ represents the cross section of a collision between two particles with initial velocities $\mathbf{v}$ and $\mathbf{v}_{*}$ and after the collision with velocities $\mathbf{v}^{\prime}$ and $\mathbf{v}_{*}^{\prime}$; and $\Omega_{\text {c.m. }}$ are all the possible dispersion angles of the collision as seen from its center of mass.

In the tetrahedral gas, the particles can travel only in four directions in three-dimensional space and all have the same absolute velocity. These directions are the ones given by joining the center of a tetrahedron with its corners. The directions can be easily viewed by recalling the directions given by a methane molecule, or equivalently, by a caltrop, which is a device with four metal points so arranged that when any three are on the ground the fourth projects upward as a hazard to the hooves of horses or to pneumatic tires (see Fig. 3).

By definition, the angle that one direction forms with any other is the same. It can be shown that the angles between different directions, $\alpha$, satisfy the relationship $\cos \alpha=-1 / 3$, which gives $\alpha=109.47^{\circ}$. The plane formed by any two directions is perpendicular to the plane formed by the remaining two directions.

We assume that the cross-section, $\sigma$, is different from zero only when the angle between the velocities of the colliding particles is $109.47^{\circ}$. It is also assumed that this collision makes the two particles leave in the remaining two directions, thus again forming an angle of $109.47^{\circ}$. A consequence of these restrictions is that the modulus of the velocity is always the same no matter how many collisions a particle has undergone and they always stay within the directions of the vertices of the tetrahedron. Furthermore, this type of gas does not break any law of physics and is perfectly valid, although hypothetical.

We label the four directions originating from the center of the caltrop with numbers, 1, 2, 3, 4 (see Fig. 3). The velocity components with the same direction but opposite sense, or equivalently, directed toward the center of the caltrop, are labeled with negative numbers $-1,-2,-3,-4$.

In order to formulate the Boltzmann equation for the tetrahedral gas, and because all directions are equivalent, we need only study the different collisions that a particle with one fixed direction can undergo. In particular if we take a particle with direction $\mathbf{- 1}$ the result of the collision with another particle with direction $\mathbf{- 2}$ are the same two particles traveling 
in directions 3 and $\mathbf{4}$; that is,

$$
(-1,-2) \rightarrow(3,4)
$$

With this in mind the last bracket of Eq. (26) is,

$$
f_{3} f_{4}-f_{-1} f_{-2}
$$

where $f_{i}$ denotes the probability of finding a particle in direction $\mathbf{i}$. Note that the dependence on velocity, $\mathbf{v}$, of the continuous velocity distribution function, $f(\mathbf{v} ; t)$, of Eq. (26) is in our case contained in the discrete subindex, $i$, of the distribution function $f_{i}$.

We can proceed in the same manner with the other remaining collisions,

$$
\begin{aligned}
& (-1,-3) \rightarrow(2,4), \\
& (-1,-4) \rightarrow(2,3) .
\end{aligned}
$$

When a particle with direction $\mathbf{- 1}$ collides with a particle with direction $\mathbf{2}$, they do not form an angle of $109.47^{\circ}$; i.e., they do not collide, they just pass by each other. This is a consequence of the previous assumption for the tetrahedral gas, which establishes a null cross section for angles different from $109.47^{\circ}$. The same can be said for collisions $(\mathbf{- 1 , 3}$, $(-\mathbf{1}, \mathbf{4})$, and $(\mathbf{- 1}, \mathbf{1})$. All these results are summarized in Table III.

Taking all this into account, Eq. (26) for direction $\mathbf{- 1}$ is reduced to a discrete sum,

$$
\frac{d f_{-1}}{d t}=\left(f_{3} f_{4}-f_{-1} f_{-2}\right)+\left(f_{2} f_{4}-f_{-1} f_{-3}\right)+\left(f_{2} f_{3}-f_{-1} f_{-4}\right),
$$

where all other factors have been set to unity for simplicity.

The seven remaining equations are:

$$
\begin{aligned}
\frac{d f_{-2}}{d t} & =\left(f_{3} f_{4}-f_{-1} f_{-2}\right)+\left(f_{1} f_{4}-f_{-2} f_{-3}\right)+\left(f_{1} f_{3}-f_{-2} f_{-4}\right), \\
\frac{d f_{-3}}{d t} & =\left(f_{2} f_{4}-f_{-3} f_{-1}\right)+\left(f_{4} f_{1}-f_{-3} f_{-2}\right)+\left(f_{1} f_{2}-f_{-3} f_{-4}\right), \\
\frac{d f_{-4}}{d t} & =\left(f_{2} f_{3}-f_{-4} f_{-1}\right)+\left(f_{3} f_{1}-f_{-4} f_{-2}\right)+\left(f_{1} f_{2}-f_{-4} f_{-3}\right), \\
\frac{d f_{1}}{d t} & =\left(f_{-3} f_{-4}-f_{1} f_{2}\right)+\left(f_{-2} f_{-4}-f_{1} f_{3}\right)+\left(f_{-2} f_{-3}-f_{1} f_{4}\right),
\end{aligned}
$$




$$
\begin{aligned}
& \frac{d f_{2}}{d t}=\left(f_{-3} f_{-4}-f_{1} f_{2}\right)+\left(f_{-1} f_{-4}-f_{2} f_{3}\right)+\left(f_{-2} f_{-3}-f_{2} f_{4}\right), \\
& \frac{d f_{3}}{d t}=\left(f_{-2} f_{-4}-f_{3} f_{1}\right)+\left(f_{-1} f_{-4}-f_{3} f_{2}\right)+\left(f_{-1} f_{-2}-f_{3} f_{4}\right), \\
& \frac{d f_{4}}{d t}=\left(f_{-2} f_{-3}-f_{4} f_{1}\right)+\left(f_{-1} f_{-3}-f_{4} f_{2}\right)+\left(f_{-1} f_{-2}-f_{3} f_{4}\right) .
\end{aligned}
$$

If we now make $f_{i}=f_{-i}(i=1,2,3,4)$ initially, this property is conserved in time. The final four equations defining the evolution of the system are:

$$
\begin{aligned}
& \frac{d f_{1}}{d t}=\left(f_{3} f_{4}-f_{1} f_{2}\right)+\left(f_{2} f_{4}-f_{1} f_{3}\right)+\left(f_{2} f_{3}-f_{1} f_{4}\right), \\
& \frac{d f_{2}}{d t}=\left(f_{3} f_{4}-f_{1} f_{2}\right)+\left(f_{1} f_{4}-f_{2} f_{3}\right)+\left(f_{1} f_{3}-f_{2} f_{4}\right), \\
& \frac{d f_{3}}{d t}=\left(f_{2} f_{4}-f_{3} f_{1}\right)+\left(f_{1} f_{4}-f_{3} f_{2}\right)+\left(f_{1} f_{2}-f_{3} f_{4}\right), \\
& \frac{d f_{4}}{d t}=\left(f_{2} f_{3}-f_{4} f_{1}\right)+\left(f_{1} f_{3}-f_{4} f_{2}\right)+\left(f_{1} f_{2}-f_{3} f_{4}\right) .
\end{aligned}
$$

Note that the ideal gas has been reduced to the tetrahedral gas, which is a fourdimensional dynamical system. The velocity distribution function, $f_{i}$, corresponds to the probability distribution function of Sec. ПI with $N=4$ accessible states.

\section{B. Evolution of the tetrahedral gas with time}

The tetrahedral gas (Eqs. 29]) reaches equilibrium when $f_{i}=1 / N$ for $i=1,2,3,4$ and $N=4$. This stationary state, $d f_{i} / d t=0$, represents the equiprobability towards which the system evolves in time. This is consistent with the previous definition of disequilibrium, Eq. (5), in which we assumed that equilibrium was reached at equiprobability, where $D=0$.

As the isolated system evolves it gets closer and closer to equilibrium. In this sense, one may intuitively think that the disequilibrium will decrease with time. In fact, it can be shown that, as the system approaches equilibrium, $D$ tends to zero monotonically with time:

$$
\frac{d D}{d t} \leq 0
$$

The analytical demonstration of this inequality for the tetrahedral gas is shown in Appendix A. 
There are even more restrictions on the evolution of this system. It would be expected that the system approaches equilibrium, $D=0$, by following the most direct path. To verify this, numerical simulations for several initial conditions have been undertaken. In all of these we observe the additional restriction that $D$ approaches $D_{\max }$ on its way to $D=0$. In fact it appears as an exponential decay of $D$ towards $D_{\max }$ in a $D$ versus $H$ plot. As an example, two of these are shown in Fig. 团, where Fig. 因(a) shows a really strong tendency towards $D_{\max }$. Contrary to intuition, among all the possible paths that the system can follow toward equilibrium, it chooses those closest to $D_{\max }$ in particular.

We can also observe this effect in a complexity, $C$, versus $H$ plot. This is shown for the same two initial conditions as the previous figure in Figure 5 .

This additional restriction to the evolution of the system is better viewed by plotting the difference $C_{\max }-C$ versus $H$. In all the cases analyzed(see two in Fig. 6) the following condition is observed:

$$
\frac{d\left(C_{\max }-C\right)}{d t} \leq 0 .
$$

This has been verified numerically and is illustrated in Figure 7, where this time derivative, which always remains negative, is shown as a function of $H$ for a grid of uniformly spaced distribution functions, $\left(f_{1}, f_{2}, f_{3}, f_{4}\right)$, satisfying the normalization condition, Eq. (3). Two system trajectories are also shown for illustrative purposes. The numerical method used to plot this function is explained in Appendix B.

\section{Maximum complexity path as an attractive trajectory}

As shown in Table $\mathbb{Z}$, a collection of maximum complexity distributions for $N=4$ can take the form

$$
\begin{aligned}
f_{1} & =f_{\max } \\
f_{i} & =\frac{1-f_{\max }}{3}, i=2,3,4
\end{aligned}
$$


where $f_{\max }$ runs from $1 / N$ (equiprobability distribution) to 1 ("crystal" distribution). The complexity of this collection of distributions covers all possible values of $C_{\max }$.

There is actually a time evolution of the tetrahedral gas, or trajectory of the system, formed by this collection of distributions. Inserting Eqs. (32) in the evolution Eqs. (29), it is found that all equations are compatible with each other and the dynamical equations are reduced to the relation,

$$
\frac{d f_{\max }}{d t}=\frac{1}{3}\left(4 f_{\max }^{2}-5 f_{\max }+1\right)
$$

This trajectory is denoted as the maximum complexity path.

Note that the equiprobability or equilibrium, $f_{\max }=1 / 4$, is a stable fixed point and the maximum disequilibrium "crystal" distribution, $f_{\max }=1$, is an unstable fixed point. Thus the maximum complexity path is a heteroclinic connection between the "crystal" and equiprobability distributions.

The maximum complexity path is locally attractive. Let us assume, for instance, the following perturbed trajectory

$$
\begin{aligned}
& f_{1}=f_{\text {max }}, \\
& f_{2}=\frac{1-f_{\max }}{3}, \\
& f_{3}=\frac{1-f_{\max }}{3}+\delta, \\
& f_{4}=\frac{1-f_{\max }}{3}-\delta,
\end{aligned}
$$

whose evolution according to Eqs. (29) gives the exponential decay of the perturbation, $\delta$ :

$$
\frac{d \delta}{d t} \sim-\left(\frac{4 f_{\max }+2}{3}\right) \delta,
$$

showing the attractive nature of these trajectories.

\section{CONCLUSION}

The time evolution of the LMC complexity, $C$, has been studied for a simplified model of an isolated ideal gas: the tetrahedral gas. In general, the dynamical behavior of this 
quantity is bounded between two extremum curves, $C_{\max }$ and $C_{\min }$, when observed in a $C$ versus $H$ phase space. These complexity bounds have been derived.

For the isolated tetrahedral gas two constraints on its dynamics are found. The first, which is analytically demonstrated, is that the disequilibrium, $D$, decreases monotonically with time until it reaches the value $D=0$ for the equilibrium state. The second is that the maximum complexity paths, $C_{\max }$, are attractive in phase space. In other words, the complexity of the system tends to equilibrium always approaching those paths. We verify this numerically; that is, the time derivative of the difference between $C_{\max }$ and $C$ is negative.

Fig. 8 summarizes the dynamical behavior of the tetrahedral gas. The different trajectories starting with arbitrary initial conditions, which represent systems out of equilibrium, evolve towards equilibrium approaching the maximum complexity path.

Whether these properties are useful in real physical systems will depend on further work on this subject. More can be said about the macroscopical nature of the disequilibrium when this work is extended to more general systems, such as to the ideal gas following the complete Boltzmann integro-differential equation. Another feature, which could result useful, would be to approximate the evolution of a real physical system trajectory to its

maximum complexity path. Note that in general, for a real system, the calculation of the maximum complexity path will not be an easy task.

\section{APPENDIX A: PROOF OF THE MONOTONICALLY DECREASE IN DISEQUILIBRIUM WITH TIME}

We now present the analytical proof of the inequality shown in Eq. (30) from Sec. IVB,

$$
\frac{d D}{d t} \leq 0
$$

The time derivative of the disequilibrium, $D$, for the isolated tetrahedral gas, is explicitly given by,

$$
\frac{d D}{d t}=\frac{d}{d t} \sum_{i}\left(f_{i}-f_{\mathrm{e}}\right)^{2}=\sum_{i} 2 f_{i} \frac{d f_{i}}{d t}-\sum_{i} 2 f_{\mathrm{e}} \frac{d f_{i}}{d t}
$$


Using the normalization Eq. (3) we find that

$$
\sum_{i} \frac{d f_{i}}{d t}=0
$$

and we are left with

$$
\frac{d D}{d t}=2 \sum_{i} f_{i} \frac{d f_{i}}{d t} .
$$

If we substitute Boltzmann Eq. (29) in the previous one, we obtain:

$$
\begin{aligned}
F \equiv \frac{1}{2} \frac{d D}{d t}= & +f_{1} f_{3} f_{4}-f_{1}^{2} f_{2}+f_{1} f_{2} f_{4}-f_{1}^{2} f_{3}+f_{1} f_{2} f_{3}-f_{1}^{2} f_{4} \\
& +f_{2} f_{3} f_{4}-f_{1} f_{2}^{2}+f_{1} f_{2} f_{4}-f_{3} f_{2}^{2}+f_{1} f_{2} f_{3}-f_{2}^{2} f_{4} \\
& +f_{1} f_{3} f_{4}-f_{3}^{2} f_{2}+f_{2} f_{3} f_{4}-f_{1} f_{3}^{2}+f_{1} f_{2} f_{3}-f_{3}^{2} f_{4} \\
& +f_{1} f_{2} f_{4}-f_{3} f_{4}^{2}+f_{1} f_{3} f_{4}-f_{2} f_{4}^{2}+f_{2} f_{3} f_{4}-f_{1} f_{4}^{2}
\end{aligned}
$$

where the new variable $F$ has been defined.

We now split this function $F$ into two different terms:

$$
\begin{gathered}
G \equiv-f_{1}^{2} f_{2}-f_{2}^{2} f_{1}-f_{3}^{2} f_{1}-f_{3}^{2} f_{2} \\
-f_{4}^{2} f_{1}-f_{4}^{2} f_{2} \\
+f_{1} f_{3} f_{4}+2 f_{1} f_{2} f_{3} \\
+2 f_{1} f_{2} f_{4}+f_{2} f_{3} f_{4}
\end{gathered}
$$

and

$$
\begin{aligned}
K \equiv \quad & -f_{3}^{2} f_{4}-f_{4}^{2} f_{3} \\
& -f_{1}^{2} f_{3}-f_{1}^{2} f_{4} \\
& -f_{2}^{2} f_{3}-f_{2}^{2} f_{4} \\
+ & f_{1} f_{2} f_{3}+2 f_{1} f_{3} f_{4} \\
+ & 2 f_{2} f_{3} f_{4}+f_{1} f_{2} f_{4} .
\end{aligned}
$$

Then 


$$
F=G+K
$$

Note that $G$ and $K$ are symmetrical functions, in the sense that we can transform one into the other by changing $\left(f_{1}, f_{2}, f_{3}, f_{4}\right)$ by $\left(f_{3}, f_{4}, f_{1}, f_{2}\right)$, respectively.

To prove that $G \leq 0$, the following variable change is performed:

$$
\begin{aligned}
& f_{1}=y_{1}, \\
& f_{2}=y_{2}, \\
& f_{3}=y_{3}+y_{4}, \\
& f_{4}=y_{3}-y_{4} .
\end{aligned}
$$

The positivity of the distributions functions, Eq. (2), implies that, $y_{1} \geq 0$ and $y_{2} \geq 0$. The function $G$ in the new variables reads,

$$
G=-y_{1}^{2} y_{2}-y_{2}^{2} y_{1}-y_{3}^{2} y_{1}-3 y_{4}^{2} y_{1}-y_{3}^{2} y_{2}-3 y_{4}^{2} y_{2}+4 y_{1} y_{2} y_{3}
$$

Regrouping terms, $G$ can be expressed as

$$
G=-y_{2}\left(y_{1}-y_{3}\right)^{2}-y_{1}\left(y_{2}-y_{3}\right)^{2}-3 y_{4}^{2} y_{1}-3 y_{4}^{2} y_{1}
$$

Since $y_{1}$ and $y_{2}$ are both positive and the squared quantities are also positive, we conclude that

$$
G \leq 0
$$

The same inequality can be demonstrated for $K$ due to its symmetry with $G$, thus proving the assumption from Eq. (A1).

\section{APPENDIX B: METHOD OF CALCULATING THE TIME DERIVATIVE OF THE MAXIMUM COMPLEXITY MINUS THE COMPLEXITY}

To calculate the quantity 


$$
\frac{d\left(C_{\max }-C\right)}{d t}
$$

from some given values of the distribution functions, $\left(f_{1}, f_{2}, f_{3}, f_{4}\right)$, we derive the expression of maximum complexity, Eq. (15), minus the definition of complexity, Eq. (11), obtaining,

$$
\frac{d\left(C_{\max }-C\right)}{d t}=\frac{d D_{\max }}{d t} H+D_{\max } \frac{d H}{d t}-\frac{d D}{d t} H-D \frac{d H}{d t} .
$$

Let us now examine each of these terms beginning from the end. The time derivative of the entropy is calculated by differentiating its definition, Eq. (6):

$$
\frac{d H}{d t}=-\frac{1}{\ln N} \sum_{i}\left[\frac{d f_{i}}{d t} \ln f_{i}+\frac{d f_{i}}{d t}\right] .
$$

But recalling Eq. (A3) we can simplify this to

$$
\frac{d H}{d t}=-\frac{1}{\ln N} \sum_{i}\left[\frac{d f_{i}}{d t} \ln f_{i}\right] .
$$

This last term can be easily calculated using the evolution Eqs. (29).

The terms $D$ and $H$ can be readily calculated using their definitions from Eqs. (5) and (6).

The time derivative of the disequilibrium has already been expressed in Appendix $\mathrm{A}$ with Eq. (A5).

The maximum disequilibrium is calculated with the previous found value of $H$ by inverting Eq. (13) and obtaining $f_{\max }$. After this, the expression defining the maximum disequilibrium, Eq. (14), can be used.

Finally the time derivative of the maximum disequilibrium is calculated as follows:

$$
\frac{d D_{\max }}{d t}=\frac{d D_{\max }}{d f_{\max }} \frac{d f_{\max }}{d H} \frac{d H}{d t}
$$

The first two derivatives on the right-hand side can be calculated analytically with Eqs. (14) and (13). The numerical value can be found using the previously calculated value of $f_{\max }$. 


\section{REFERENCES}

[1] C. H. Bennet, Found. Phys. 16, 585 (1986).

[2] J. P. Crutchfield and Karl Young, Phys. Rev. Lett. 63, 105 (1989).

[3] G. Chaitin, J. ACM 13, 547 (1966).

[4] A. N. Kolmogorov, Dokl. Akad. Nauk. SSSR 119, 861 (1958).

[5] J. G. Sinai, Dokl. Akad. Nauk. SSSR 124, 768 (1959).

[6] S. Lloyd and H. Pagels, Ann. Phys. (N.Y.) 188, 186 (1988).

[7] P. Grassberger, Int. Journal Theor. Phys. 25, 907 (1986).

[8] J. S. Shiner, M. Davison, and P. T. Landsberg, Phys. Rev. E 59, 1459 (1999).

[9] P. T. Landsberg and J. S. Shiner, Phys. Lett. A 245, 228 (1998).

[10] H. Atmanspacher, C. Räth, and G. Wiedermann, Physica A 234, 819 (1997).

[11] R. López-Ruiz, H. L. Mancini, and X. Calbet, Phys. Lett. A 209, 321 (1995).

[12] D. P. Feldman and J. P. Crutchfield, Phys. Lett. A 238, 244 (1998).

[13] M. Gell-Mann, Complexity 1, 16 (1995).

[14] V. Latora and M. Baranger, Phys. Rev. Lett. 82, 520 (1999).

[15] C. Anteneodo and A.R. Plastino, Phys. Lett. A 223, 348 (1996). 


\section{FIGURES}

FIG. 1. Maximum, minimum, and minimum envelope complexity, $C_{\max }, C_{\min }$, and $C_{\operatorname{minenv}}$ respectively, as a function of the entropy, $H$, for a system with $N=4$ accessible states.

FIG. 2. Maximum, minimum, and minimum envelope disequilibrium, $D_{\max }, D_{\min }$, and $D_{\operatorname{minenv}}$ respectively, as a function of the entropy, $H$, for a system with $N=4$ accessible states.

FIG. 3. The four possible directions of the velocities of the tetrahedral gas in space. Positive senses are defined as emerging from the center point and with integer numbers, $1,2,3,4$.

FIG. 4. Time evolution of the system in $(H, D)$ phase space for two different initial conditions at time $t=0$ : (a) $\left(f_{1}, f_{2}, f_{3}, f_{4}\right)=(0.8,0.2,0,0)$ and (b) $\left(f_{1}, f_{2}, f_{3}, f_{4}\right)=(0.5,0.5,0,0)$. The maximum and minimum disequilibrium are shown by dashed lines.

FIG. 5. Time evolution of the system in $(H, C)$ phase space for two different initial conditions at time $t=0$ : (a) $\left(f_{1}, f_{2}, f_{3}, f_{4}\right)=(0.8,0.2,0,0)$ and (b) $\left(f_{1}, f_{2}, f_{3}, f_{4}\right)=(0.5,0.5,0,0)$. The maximum and minimum complexity are shown by dashed lines.

FIG. 6. Time evolution of the system in $\left(H, C_{\max }-C\right)$ phase space for two different initial conditions at time $t=0$ : (a) $\left(f_{1}, f_{2}, f_{3}, f_{4}\right)=(0.8,0.2,0,0)$ and (b) $\left(f_{1}, f_{2}, f_{3}, f_{4}\right)=(0.5,0.5,0,0)$. The values $C_{\max }-C_{\min }$ are shown by dashed lines.

FIG. 7. Numerical verification of $d\left(C_{\max }-C\right) / d t \leq 0$. This time derivative is shown as a function of $H$. A grid of uniformly spaced, $\Delta f_{i}=0.5$, distribution functions, $\left(f_{1}, f_{2}, f_{3}, f_{4}\right)$, satisfying the normalization condition Eq. (3), have been used. Two system trajectories for initial conditions, $t=0,\left(f_{1}, f_{2}, f_{3}, f_{4}\right)=(0.8,0.2,0,0)$ and $\left(f_{1}, f_{2}, f_{3}, f_{4}\right)=(0.5,0.5,0,0)$ are also shown for illustrative purposes. It can be seen how the above-mentioned time derivative always remains negative. 
FIG. 8. Summary of this paper. The time evolution of the system for three different initial conditions, $t=0,\left(f_{1}, f_{2}, f_{3}, f_{4}\right)=(0.8,0.2,0,0),\left(f_{1}, f_{2}, f_{3}, f_{4}\right)=(0.5,0.5,0,0)$, and the maximum complexity path are shown. The minimum complexity is shown by dashed lines. It can be seen how the system tends to approach the maximum complexity path as it evolves in time toward equilibrium. 


\section{TABLES}

TABLE I. Probability values, $f_{j}$, that give a maximum of disequilibrium, $D_{\max }$, for a given $H$

\begin{tabular}{ccc}
\hline \hline $\begin{array}{c}\text { Number of states with } \\
\text { with } f_{j}\end{array}$ & $f_{j}$ & Range of $f_{j}$ \\
\hline 1 & $f_{\max }$ & $\frac{1}{N} \ldots 1$ \\
$N-1$ & $\frac{1-f_{\max }}{N-1}$ & $0 \ldots \frac{1}{N}$ \\
\hline \hline
\end{tabular}

TABLE II. Probability values, $f_{j}$, that give a minimum of disequilibrium, $D_{\min }$, for a given $H$

\begin{tabular}{ccc}
\hline \hline $\begin{array}{c}\text { Number of states } \\
\text { with } f_{j}{ }^{\text {a }}\end{array}$ & $f_{j}$ & Range of $f_{j}$ \\
\hline$n$ & 0 & 0 \\
1 & $f_{\min }$ & $0 \ldots \frac{1}{N-n}$ \\
$N-n-1$ & $\frac{1-f_{\min }}{N-n-1}$ & $\frac{1}{N-n} \cdots \frac{1}{N-n-1}$ \\
\hline \hline
\end{tabular}

${ }^{a} n$ can have the values $0,1, \ldots N-2$

TABLE III. Cross sections, $\sigma$, for a particle in direction $\mathbf{- 1}$ colliding with particles in the other remaining directions of the tetrahedral gas

\begin{tabular}{cc}
\hline \hline Collision & Cross section \\
& $\sigma$ \\
\hline$(-\mathbf{1},-\mathbf{2}) \rightarrow(\mathbf{3}, \mathbf{4})$ & 1 \\
$(-\mathbf{1},-\mathbf{3}) \rightarrow(\mathbf{2}, \mathbf{4})$ & 1 \\
$(\mathbf{- 1},-\mathbf{4}) \rightarrow(\mathbf{2}, \mathbf{3})$ & 1 \\
Other collisions & 0 \\
\hline \hline
\end{tabular}

